\title{
Über die elektrometrische Messung der Reaktion organischer Flüssigkeiten.
}

Von

Carlo Fos aus. Turin.

In dem am 8. Oktober 1906 erschienenen Hefte dieses Archives wurden mehrere Arbeiten von Prof. Tangl und seinen Schülern ${ }^{1}$ ) über die Bestimmung der Hydrogenionen-Konzentration des Magensaftes, des fötalen Blutes und des Blutes bei Diabetes veröffentlicht.

Obwohl diese Arbeiten mehrere Monate nach meiner Arbeit im Archivio di Fisiologia ${ }^{2}$ ) erschienen sind, wird letztere von den A u toren nicht berücksichtigt. Es ist aber wahrscheinlich, dass das so verbreitete Archivio di Fisiologia auch dem physiologisch-chemischen Institut in Budapest zur Verfügung steht.

Meine Untersuchungen hatten mich zu einer Modifikation der bestehenden Methoden und zur Konstruktion eines neuen Apparates geführt. Dieser macht es möglich, eine elektrometrische Bestimmung des Hydrogenionengebaltes in einer kleinen Menge Flüssigkeit und in wenigen Minuten auszuführen und hat dabei gegen die Methoden von Höber, Farkas, Fraenkel und Pfaundler-Szili, welche letztere von Tangl und seiner Schule angewandt wurden, den Vorteil, eine einzige leicht und rasch einstellbare Gaselektrode genügend zu machen.

Durch dieselbe ist auch die Bereitung einer isohydrischen Lösung überflüssig geworden. Die Resultate, die Prof. Tangl bei dem

1) F. Tangl, Untersuchungen über die Hydrogenionenkonzentration im Inhalte des nüchternen menschlichen Magens. Pflüger's Arch. Bd. 115 S. 64. A. Szili, Untersuchungen über den Hydroxylionengehalt des plazentaren (fötalen) Blutes. Pflüger's Arch. Bd. 115 S. 72. - H. Benedikt, Der Hydroxylionengehalt des Diabetikerblutes. Pflù ger's Arch. Bd. 115 S. 106.

2) C. Foā, La reazione dei liquidi dell' organismo determinata col metodo elettrometrico (pile di concentrazione). Arch. di Fisiologia vol. 3 p. 369-415. Marzo 1906. 
menschlichen Magensafte erreichte, stimmen mit denjenigen überein, die ich bei dem Safte des Hundes und des Menschen (ein Fall) schon erreicht hatte, und bestätigen auch, was ich über die Anwendung der verschiedenen Indikatoren bei den Magensaft-Untersuchungen berichtet habe.

Die Untersuchungen von $\mathrm{H}$. Benedikt über die Reaktion des Blutes bei Diabetikern sind eng mit denjenigen verknüpft, die Mme Gatin-Gruczewska und ich über die Blut- und Harnreaktion bei den Säureautointoxikationen während der experimentellen Glykosurien ausgeführt haben. Das Ergebnis dieser Untersuchungen war folgendes: Bei dem Stichdiabetes, sowohl des Hundes als des Kaninchens, erleidet das Blut nur eine leichte und vorübergehende Steigerung der Azidität, während der Harn stark sauer wird; bei dem Adrenalindiabetes dagegen wird das Blut stark sauer, während der Harn nur leicht seine Reaktion verändert. In der Arbeit von Dr. H. Benedikt ist nicht angegeben, um was für einen Diabetes es sich in den von ihm untersuchten Fällen handelte, und es wäre auch wünschenswert gewesen, die Reaktion des Harnes zu untersuchen, welche am leichtesten bei der Glykosurie beeinflusst wird. 